\title{
Antitumor Drug Mitoxantrone Do Not Preclude Salt-driven B-Z Transition of Poly (dG- dC)
}

\author{
Armen T. Karapetyan", Margarita A. Torosyan, Yurik S. Babayan \\ Department of Physics and Electrical Engineering, National University of Architecture and Construction of Armenia, Armenia
}

Copyright $\bigcirc 2015$ by authors, all rights reserved. Authors agree that this article remains permanently open access under the terms of the Creative Commons Attribution License 4.0 International License

\begin{abstract}
Despite of the existence in literature of large number of reports the binding modes of mitoxantrone (MTX) with DNA must be subject to refinement. Moreover there is no any report yet on interaction of MTX with Z-DNA. Here we present results of circular dichroism (CD) studies on MTX binding to salt driven left-handed conformation of poly (dG- dC). It was shown, that in contrast with classical intercalating ligands (ethidium bromide, acridine orange etc.) that bind with B-form DNA and obstacle B-Z transition at high concentrations of $\mathrm{NaCl}$ MTX interferes B-Z transition, but does not prevent the transitions process, thus suggest the semi intercalating (partial stacking) mode of MTX interaction with Z-form of DNA.
\end{abstract}

Keywords Mitoxantrone, Complexes MTX-poly(dG-dC), B-Z Transition of Complexes, Semi Intercalation

\section{Introduction}

Small molecules including anticancer drugs and antibiotics (ligands) correlate their biological and therapeutic activities with the ability of interaction with existence in living cells of various DNA conformations (B, A, Z, cruciform, etc.) [1-6].

Discovery of existence of unusual DNA conformations (Z-DNA, cruciform, bent DNA, oligopurine oligopyrimidine structures, etc.), in living cells and intense study of their interaction with biological active ligands made it possible to presume that alternative structures could have a functional role in vivo [1-4,7,8]. Z-DNA, a left-handed double helix particularly, may play positive or negative regulatory role in the transcriptional process. It is likely that part of these activities will be the result of specific interactions of the ligands with Z-DNA.

Many studies have been carried out describing Z-DNA altered reactivity with carcinogens. A number of ligands (proteins, antibodies, drugs) have been shown to influence the B-Z equilibrium in solution [2, 8-10]. Mitoxantrone (MTX), a member of the anthracycline antibiotics, is one of most intensive studied drugs. Recent enhanced interest in the
MTX has arisen because of its apparent lower risk of cardiotoxic effects compared with the naturally occurring anthracycline drugs [9].

MTX has a planar anthraquinone ring and two nitrogen-containing side chains (Fig. 1) [9]. The interaction of MTX with DNA has been examined by various biochemical and physicochemical methods ([11] and reference in it). It was recognized that MTX realizes its biological activity by complexing with DNA. The mode of interaction has been established to be intercalation, groove banding and electrostatic binding with B-form of DNA [9-13].

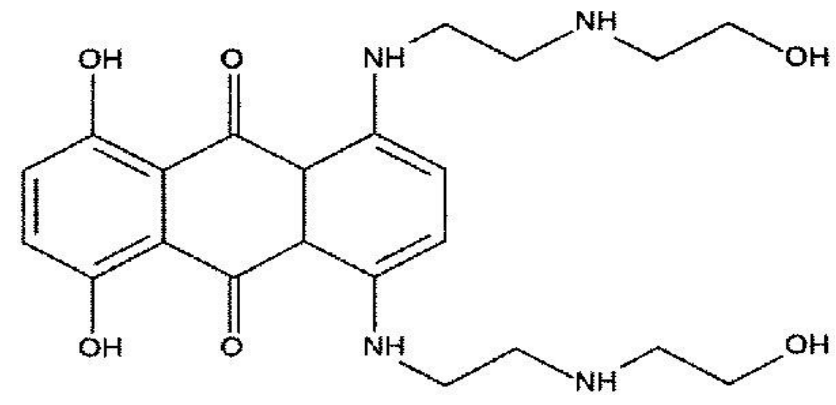

Figure 1. Chemical Structure of mitoxantrone (MTX)

Recent in vitro [2-4] and in vivo [3,5] discoveries support the concept that DNA is a conformational dynamic molecule which plays an active role in gene expression. Particularly it was demonstrated that certain segments of DNA exist in a balance between B- and Z-helices and the B-to Z-transition is influenced by environmental factors (ionic conditions, protein binding, drugs, or carcinogens) $[1-2,8,14,15]$. Current literature data shows that there is no any report yet about the detection of MTX interaction with Z-DNA. To full this gap we investigate the binding affinity of MTX with salt driven Z-form of poly (dG-dC).

Obtained experimental data for the first time report that the increase of mitoxantrone concentration causes slight changes on the CD spectra of Z-form of poly (dG-dC), which considered as evidence of weak interaction of MTX with the $\mathrm{Z}$ form of polymer. In contrast with the other intercalating to ds-DNA structure ligands that bind with B-form DNA and obstacle B-Z transition at high concentrations of $\mathrm{NaCl}$, 
mitoxantrone interferes B-Z transition, but does not cause a barrier the transitions process despite of ligand concentration in solution.

\section{Material and Methods}

Calf thymus DNA (CT), poly (dG- dC) provided from sigma, MTX was provided from Farmitia (Italy). Circular dichroic (CD) spectra were recorded by Jobin Ivon Mark III dichrograph in a thermostated cell. Since transition between $\mathrm{B}$ and $\mathrm{Z}$ forms is slow (from several minutes up to several hours depending on conditions: ionic strength, temperature), special care was applied to reveal observed change reached saturation. Thus, all the data presented herein pertain to equilibrium conditions.

The measurements were made at $25{ }^{\circ} \mathrm{C}$ in buffer $0.1 \mathrm{M}$ $\mathrm{NaCl}, 0.01 \mathrm{M}$ Tris $\mathrm{HCl}, 0.01 \mathrm{mM}$ EDTA at $\mathrm{pH}=7.4$. Tris ETDA and $\mathrm{NaCl}$ were Serva products. Concentrations of the samples were found using the following molar extinction coefficients, in $\mathrm{M}^{-1} \mathrm{~cm}^{-1}: \varepsilon_{260}(P)=6550$ for CT DNA, $\varepsilon_{254}(P)=7100$ for poly $(\mathrm{dG}-\mathrm{dC}), \varepsilon_{659}=25900$ for MTX and $\varepsilon_{480}=5850$ for ethidium bromide (EtBr). The absorption spectra were obtained with SPECORD UV VIS spectrophotometer. Quartz cells with the optical path of $1 \mathrm{~cm}$ were employed. In all optical measurements in which changes in the spectrum of DNA and poly $(\mathrm{dG}-\mathrm{dC})$ were recorded as a function of MTX concentration, nucleic acids concentration was maintained constant by addition of MTX solution containing polymer at the same concentration as that contained in the cell.

The studies conducted showed that MTX molecules are optically inactive (which was to be expected, since the molecules are symmetrical) and do net become optically active upon interaction with nucleic acids. The latter interaction changed the intensities and the positions of bands in circular dichroic spectra only in the region of the optical activity of nucleic acids.

\section{Results and Discussion}

Several in vitro and in vivo experiments revealed the existence of unusual conformation of DNA, including left-handed DNA (Z-DNA) cruciform, bent DNA, oligopurine-oligopyrimidine parallel structures [4-11]. The possibility of structural transition in DNA depending on the environmental conditions has been convincingly demonstrated before [3-5]. Sequence motives, which may adopt unusual DNA structures in the vicinity of many non-coding regions of genome, indicate that such structures including left-handed helices may play important role in regulatory processes [16-17]. On the other hand it is well established that many small biological active compounds (ligands) including anticancer drugs and antibiotics, realize their therapeutic activities with the ability of intercalative interaction with DNA [18]. Experimental studies showed that prominent representatives of intercalators such as ethidium bromide, actinomicine $\mathrm{D}$ and many others [19-21] stabilize B-form of DNA and inhibit B-Z transition. According to published experimental data the dominant mode of antibiotic mitoxantrone (MTX) with DNA is intercalation which radically differ from that of known for EtBr, AMD and others [8-11]: the enthalpy of MTX binding with ds-DNA is $-(2 \div 3) \mathrm{kcal} / \mathrm{mol}$, which much smaller than that of for classical intercalators [13, 14]. This type of interaction is recognized as semi intercalation [11-12]. In order to answer the question "Is the mechanism of semi intercalator MTX binding with Z-DNA like that of EtBr and other classical intercalators?" we investigate the interaction of MTX with driven by $3.1 \mathrm{M} \mathrm{NaCl} Z$-form of $\operatorname{poly}(\mathrm{dG}-\mathrm{dC})[1]$.

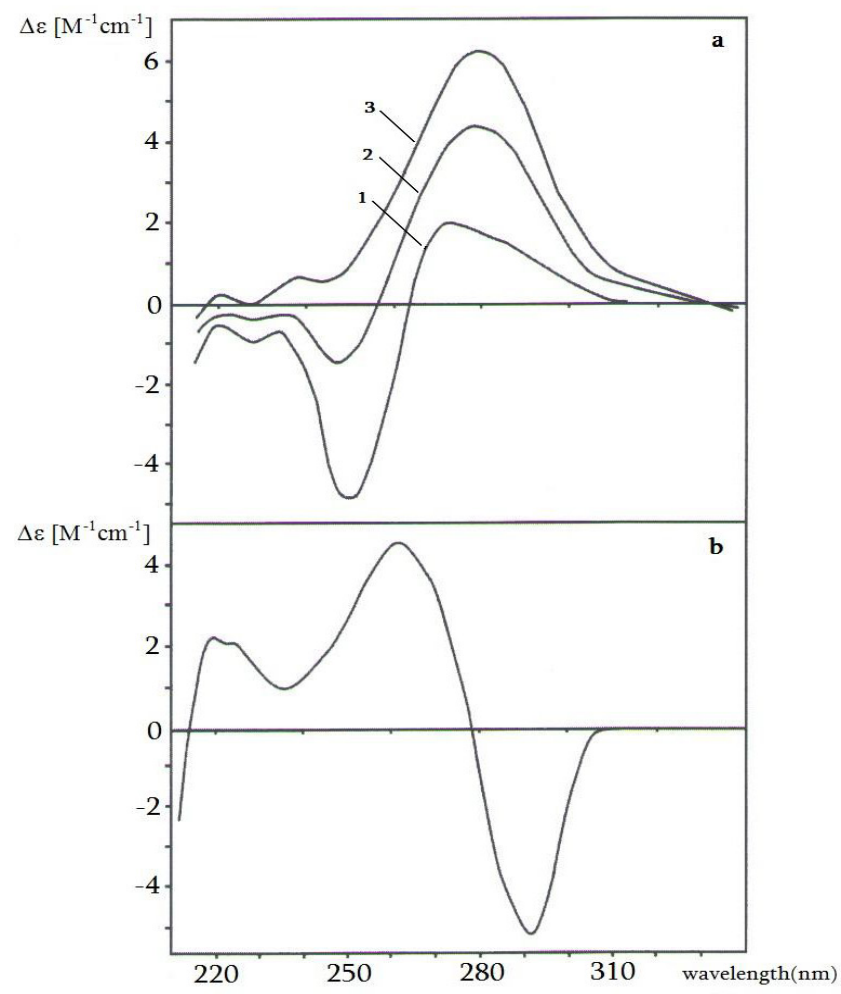

Figure 2. Circular dichroic spectra of MTX-poly (dG-dC) complexes at $\mathrm{pH} 7$ and $0.1 \mathrm{MNaCl}$ containing $\mathrm{P}=510^{-5} \mathrm{M}$ b.p. and $\mathrm{D} / \mathrm{P}=0(1) ; 0.16$ (2) and 2.1(3)(a) and at the same D/P mention as in (a) and 3.1 $\mathrm{M} \mathrm{NaCl}$ (b). D and $\mathrm{P}$ are the concentrations of MTX and DNA base pairs respectively. All experiments have been conducted at $25^{\circ} \mathrm{C}$.

The interaction of MTX with poly (dG-dC) was studied by circular dichroism (CD) spectroscopy at different drug to DNA base pair ratios $((\mathrm{D} / \mathrm{P}=\mathrm{r})$, where $\mathrm{D}$-is the molar concentration of ligand and P-molar concentration of base pairs of DNA). The CD spectrum of pure poly (dG-dC) sample at ultraviolet region is typical for B-conformation of the polynucleotide having a maximum at $275 \mathrm{~nm}$ and a negative band at $250 \mathrm{~nm}$ (curve 1, Figure 2a).

These bands are considered as marker bands for B-conformation of DNA [33-35]. Thus, conformational transition of DNA could be traced by changes in the positions and intensity of these spectral bands in CD spectra. Addition 
of the ligand to the purine-pyrimidine solution raised unusual and significant reversal of the negative $250 \mathrm{~nm}$ band at the saturation of the binding sites ligand concentrations $(\mathrm{r}=2.1)$, it become positive (cp. curves 1 and 3, Figure 2a). Registered induction of $C D$ in the 250 and $278 \mathrm{~nm}$ region suggests that MTX intercalates to the polynucleotide helix though the mode of intercalation is different from that of EtBr and other strong stabilizers of B-DNA [28]. Increase of the concentration of such ligands in solution of poly $(\mathrm{dG}-\mathrm{dC})$ of Z-form (high salt form of polymer) displace the Z-B equilibrium in favors of B-form [1], and the CD spectrum of the polymer become similar to that of B-form [29]. As follows from the Figure $2 \mathrm{~b}$, regardless of the concentration of MTX, in solution containing 3.1 M NaCl, poly (dG-dC) stored in the Z-form. To argue against the assumption that interaction is due to high salt concentration $(3,1 \mathrm{M} \mathrm{NaCl})$ the experiments with the complex of MTX and CT DNA at 3,1M $\mathrm{NaCl}$ (at this salt concentrations CT DNA is in B-form) have been performed. Figure 3 shows the CD spectra of free CT DNA (curve 1) and its complexes with MTX (curves 2, 3). Registered negative band at $243 \mathrm{~nm}$ and two positive bands at $223 \mathrm{~nm}$ and $277 \mathrm{~nm}$ in the CD spectrum of free CT DNA are considered as the B-conformation marker bonds [30, 31]. Upon the interaction of MTX with CT DNA these bands do not show any appreciable shifts (cp. curves 1, 2 and 3, Figure 3 ), indicating that DNA remains in native B-conformation [30-31]. Observed increase in intensity of the spectrum of complexes has been attributed to local perturbation of native B conformation of DNA helix [31, 32]. Therefore the conservative form of CD spectrum of the complexes of MTX with high-salt form of poly (dG-dC) (Figure $2 \mathrm{~b}$ ) might be assigned to high salt L-form of the polynucleotide but not the high-salt concentration itself in solution, respectively, which may be responsible for noted weak induced CD at the $223 \mathrm{~nm}$ and $272 \mathrm{~nm}$ bands in the spectrum of the complex of MTX with poly (dG-dC) (cp. curve 2 with curve 1, Figure 4).

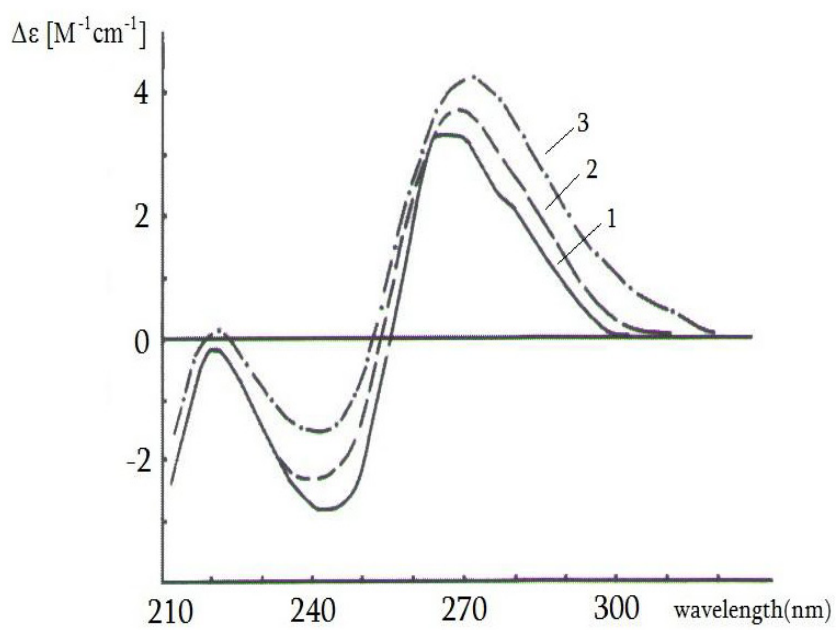

Figure 3. Circular dichroic spectra of CT DNA ( $\mathrm{P}=8.2 \cdot 10^{-5} \mathrm{M}$ b.p. $)$ at $3.1 \mathrm{M} \mathrm{NaCl}$ and $\mathrm{D} / \mathrm{p}=0(1) ; 0,09$ (2) and 0.25 (3). $\mathrm{pH}=7,0$. All experiments have been conducted at $25^{\circ} \mathrm{C}$.
The possible interpretation of the binding data at high-salt concentration of the complexes MTX-poly $(\mathrm{dG}-\mathrm{dC})$ is that MTX bind only weakly to the Z-form but not strongly (like EtBr) to the B-form of polynucleotide which presumably is the different mode of intercalation from that of $\mathrm{EtBr}$ or acridine orange. The existence of the induced CD (ICD) in the region 250 and $278 \mathrm{~nm}$ at $\mathrm{UV}$ and $688 \mathrm{~nm}$ at visible region [28] where only the ligand but not DNA absorbs made it possible to suggest that two symmetrical chains of MTX (Figure 1) prevent the classical type of intercalation. According to the results of NMR experiments the mode of interaction of MTX with DNA is likely semi intercalation which presumably may be the source of ICD [28]. The effect of this type of intercalation quite differs from that of EtBr. The latter strongly binds to the B-form of DNA and shifts the conformational equilibrium to the B-form. Therefore the structure of complexes of the EtBr-poly (dG-dC) at saturating concentration of the ligand is the same at high-or low salt concentrations. This assumption is supported by the experiment which demonstrates that addition of $3.1 \mathrm{M} \mathrm{NaCl}$ to the solution of the EtBr-poly $(\mathrm{dG}-\mathrm{dC})$ complexes do not cause conformational change (Figure 4, curve 1): after 40 min the structure of complexes remains at B-form. It is well established that when the salt concentration is increased up to or more that $2.5 \mathrm{M} \mathrm{NaCl}$ polynucleotide poly $(\mathrm{dG}-\mathrm{dC})$ in a well redistricted way changes from B-form (low salt R-form) to the high salt L-form (Z-form) [1, 2], which is characterized particularly by inversion of the CD spectrum at $295 \mathrm{~nm}$ (compare curves 1, Figure 2a and 2b). Our experimental data show that presence of MTX in solution slow down the B-Z transition in time with respect of pure high-salt form of polynucleotide (compare curves 1 and 2, Figure 5) which points to the ability of MTX preclude, but not prevent (like EtBr, curve 3, Figure 5) low salt R-form to the high-salt L-form poly $(\mathrm{dG}-\mathrm{dC})$ transition.

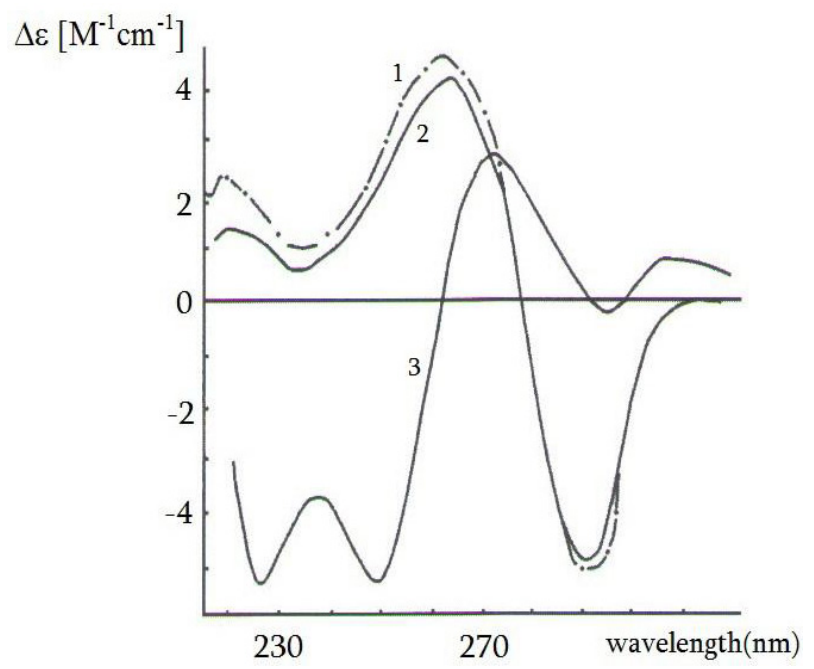

Figure 4. Circular dichroic spectra of $\mathrm{P}=5 \cdot 10^{-5} \mathrm{M}$ b.p. pure poly (dG-dC) (1) and its complexes with MTX (2) and $\mathrm{EtBr}(3)$ after adding 3.1 MNaCl. $\mathrm{D} / \mathrm{P}=0,25$ for complexes MTX and $\mathrm{EtBr}$. 


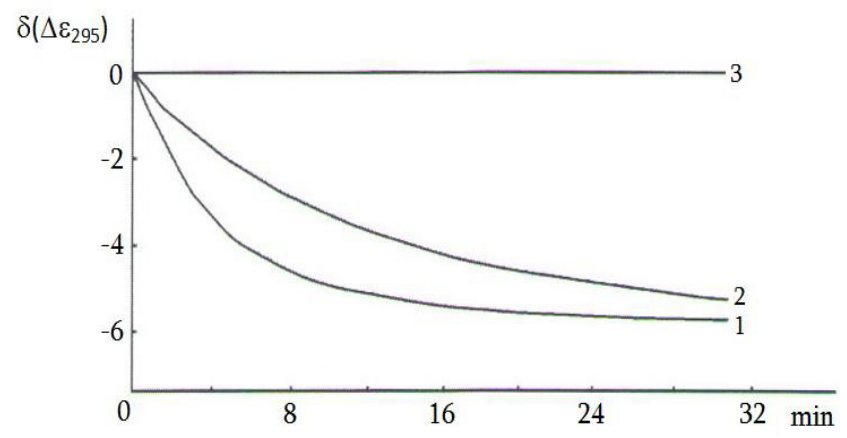

Figure 5. Time-dependent change of $\mathrm{B}-\mathrm{Z}$ transition for pure poly (dG-dC) (1); MTX-poly (dG-dC) (2) and EtBr-poly (dG-dC) (3) complexes. Conditions are the same as in Figure 4.

Experimental results presented above made it possible to postulate that interaction of MTX with DNA is not similar to the intercalation of $\mathrm{EtBr}$, daunomycin, acridine orange and etc. By contrast, MTX interacting with B-DNA precludes the $\mathrm{B}-\mathrm{Z}$ transition but unlike classical intercalation does not prevent the conversion B-form of DNA of high salt concentrations, to the Z-form of DNA. This finding extends the picture of MTX binding to different DNA forms and may enhance the suitability of the ligand as a model for the design of structure specific DNA binding analogs.

\section{REFERENCES}

[1] Rich A., Hordheim A. and Wang A.H.J. The chemistry and biology of the left-handed Z-DNA. Aim. Rev. Biochem. $1984,51,791-846$.

[2] Jovin T. M., McIntosh L.P., Arndt-Jovin D. J., Zarling D.A., Nicond M. R., van de Sand J.H., Jorgenson K.E. and Ecksten F. Left-handed DNA: from Synthetic Polymers to Chromosomes. J. Biomol Struct. Dyn 1983,1, 21-125.

[3] Brahmachari S. K., Ramesh N., Shouche Y. S., Mishra R. K., Bogga R. and Meera Q., Unusual DNA structures: Sequence Requirements and Role in Transcriptional Control. Structure and Methods. v. 2. DNA Protein Complexes and Protein. (Eds. R. H. Sarma and M. H. Sarma, Adenine Press 1990, 33-49).

[4] Wells R.D. and Harvey S.C. Unusual DNA Structures. Springer, New York, 1987

[5] Nordheim A. Z-DNA: Exploring the Biological Significance Nucleic Acids and Molecular Biology v.1 (ed. F Eckstein end D.M.J. Lilly) Springer - Verlag Berlin Heidelberg 1987, $112-125$

[6] Saenger W., Principles of Nucleic Acid Structure, 1984, Springer-Verlag, New York.

[7] Jaworski A., Hsien W-H, Blaho J.A., Larson J. E. and Wells R.D., Left-Handed DNA in Vivo, Science,1987, 38, 773-777.

[8] Zacharias W., Jaworski A., Larson J. E. and Wells R.D., The B-to Z-DNA equilibrium in vivo is perturbed by biological processes, Proc. Natl. Acad. Sci. USA, 1988, 85, 7069-7073.

[9] Kapuscinski J., Darzynkiwic Z., Traganos F., Melamed M. R., Interaction of a new antitumor agent, 1,4-dihy- droxy-5,8-bis [[2-[(2-hydroxyethyl) amino]-ethyl]amino]-9,10- anthracene dione, with nucleic acids, Biochem Parmacol. 1981, 31,231-240.

[10] Li N., Ma Y, Yang Ch., Guo L. and Yang X., Interaction of anticancer drag mitoxantrone with DNA analyzed by electrochemical and spectroscopic methods. Biophysical Chemistry 2005, 116,199-205.

[11] Awathi P., Dogra Sh. and Barthwal R., Multispectroscopic methods of interaction of anticancer drug mitoxantrone with Poly (dG-dC). Poly (dG-dC) and Poly (dA-dT). Poly (dA-dT). J. Phatochem. Phatobiol. B. Biology. 2013, 127, 78-87.

[12] C. Panousis, D. R. Phillips, DNA sequence specificity of mitoxantrone, Nucleic Acids Res. 22 (1994) 1342-1345.

[13] J.W. Lown, A. R. Morgan, S.F. Yen, Y.H. Wang, W.D. Wilson, Characteristics of the binding of the anticancer reagents mitoxantrone and ametantrone and related structures to deoxyribonucleic acids, Biochemistry 24 (1985) 4028-4035.

[14] Karapetyan A. T, Minyat E. E and Ivanov V. I., Increase in temperature induces the B-Z transition of poly $d(\mathrm{G}-\mathrm{G})$ in water - ethanol solution, FEBS Lett., 173, 243-246, 1984.

[15] Ivanov V. I., Minyat E. E, The transitions between left-and right-hand d forms of poly (dG-dC), Nucieic Acias Research, 4783-4798, 1981.

[16] Qasha P.K., Safaya S.K., Similarity of the nucleotide sequences of rat a-lactalbumin and chicken lysozyme genes, 1984, Nature 308, 377-308.

[17] V.I. Lyamichev, S. M. Mirkin, O. N. Danilevskaya, O. N. Voloshin, S. V. Balatskaya, V. N. Dobrynin, S.A. Filippov \& M. D Frank-Kamenetskii, An unusual DNA structure detected in a telomeric sequence under superhelical stress and atlow pH, Nature 339, 634, 1989.

[18] Waring M.J., The molecular Bases of Antibiotic Action. John Wiley and Sons N, Y, 1972.

[19] Walker G. T., Stone M. P. Krugh T. R. Ethidium binding to left-handed $(\mathrm{Z}) \mathrm{DNA}_{\mathrm{s}}$ results in regions of right- handed DNA at intercalation site // Biochemistry, 1985, v.24, N24, p. 74627471 .

[20] Winkle S.A., Krugh T. R. Equilibrium binding of carcinogens and antitumor antibiotics to DNA site selectivity, cooperativity, allosterism //Nucl. Acids Res., 1981, v.9, N13, p. 3175- 3186 .

[21] Van de Sande J. H., Jovin T. M. Z* DNA, the left handed helical form of poly [d(G-C)] in $\mathrm{MgCl}$-ethanol, is biologically active //EMBO J., 1982, v. 1, N 1, p. 115-120.

[22] Zimmer C., Marek C., Gushlbauer W. Z-DNA and other non-B-DNA structures are reversed to B-DNA by interaction with netropsin //FEBS Lett., 1983, v.1 54, N1, p. 156-160.

[23] Chaires J.B. Daunomycin inhibits the $\mathrm{B} \rightarrow \mathrm{Z}$ transition in poly [d(G-C)] //Nucl. Acids Res., 1983, v.1 1, N23, p. 8485-8494.

[24] Lown J. W., Morgan A. R. Yen S.-F., Wang Y.-H., Wilson W.D. Characteristics of the binding of anticancer agents mitoxantrone and ametantrone and related structures to deoxyribonucleic acids // Biochemistry, 1985, v.24, N15, p. 4028-4035. 
[25] Babayan Yu, Manzini G., Xodo L.E., Quadriofoglio F., Base specificity in the ethidium with synthetic polyribonucleotides // Nucleic Acids Res., 1987, v. 155812.

[26] Kotovych G., Lown J. W., Tong J. P. K. High-field 'H and ${ }^{31} \mathrm{P}$ NMR studies on the binding of the anticancer agent mitoxantrone to $d\left(\mathrm{C}_{\mathrm{p}} \mathrm{GpApT} \mathrm{T}_{\mathrm{p}} \mathrm{CpG}\right) 2$ // J. Biomol. Struc. Dyn. 1986, v.4, N1, p.1 11-125.

[27] Awasti P., Dogra S., Barthwal R. Multispectroscopic methods reveal different modes of interaction of anticancer drug mitohantrone with poly (dG-dC)- poly $(\mathrm{dG}-\mathrm{dC})$ and poly(dA-dT) . J. Photochem. Photobiol. B: Biology, 2013, v. 127, p. $78-87$

[28] Rao M.V.R., Atreyi M. and Kunar A., Interaction of antitumor Agent mitoxatrone with poly $[\mathrm{d}(\mathrm{G}-\mathrm{C})]$-a circular dicroic Study. Indian Journal of Biochemistry and biophysics. 26, 5-8,
1989.

[29] Pohl F.M., Jovin T.M., Baehr W., Holbrook J.J. Ethidium bromide as a cooperative effector of a DNA structure //Proc. Nat. Acad. Sci. USA.-1972, v.69., N12., p.3805-3809.

[30] M. Vorlickova, Conformational transitions of alternating purine-pyrimidine DNAs in perchlorate ethanol solutions, Biophys J. 69, 1995, 2033-2043.

[31] J. Kypr, M. Vorlickova, Circular dichroism spectroscopy reveals invariant conformation of guanine runs in DNA, Biopolymers (Biospectroscopy) 67, 2002, 275-277.

[32] D.K. Jangir, S. K. Dey, S. Kundu, R. Mehrotra, Assessment of amsacrine binding with DNA using UV-Visible, circular dichroism and Raman spectroscopic techniques,]. Photochem. Photobiol. B: Biol. 114, 2012, 38-43. 